\title{
Iterative Size Reduction Technique for Optical Nanostructures
}

\author{
T. Khudiyev ${ }^{1,2, *}$, M. Bayindir ${ }^{1,2,3}$ \\ ${ }^{1}$ National Nanotechnology Research Center, Bilkent University, Ankara 06800, Turkey \\ ${ }^{2}$ Institute of Materials Science and Nanotechnology, Bilkent University, Ankara 06800, Turkey \\ ${ }^{3}$ Department of Physics, Bilkent University, Ankara 06800, Turkey \\ *e-mail: khudiyev@bilkent.edu.tr
}

\begin{abstract}
One-dimensional optical nanostructures are mass produced from a macroscopic semiconductor rod by using a new top-to-bottom fabrication approach. These nanostructures, which can be easily scaled up to 2D and 3D, exhibit sizedependent double coloration mechanisms.

OCIS codes: (220.4241) Nanostructure fabrication; (290.4020) Mie theory; (310.6628) Subwavelength structures, nanostructures
\end{abstract}

Light material interactions at the nanoscale are being extensively studied in nanowires and other 1D nanomaterials due to their unique resonant absorption and scattering abilities. Light confinement, leaky-mode resonances, and non resonant interference effects can be used to tune and enhance light absorption in a wide range of high performance, nanowire-based photonic and optoelectronic devices, including lasers, solar cells, chemosensors, and photodetectors. However these examples are exclusively for single dielectric or semiconducting nanowires or their arrays that cannot be easily scaled up for mass production.

Here, we report fabrication of large-area 1D optical nanostructures obtained by a novel thermal size reduction technique. Nanowire fabrication by thermal drawing is based on size reduction of thermally compatible materials such as high temperature engineering polymers, low melting temperature chalcogen glasses, and low melting temperature metals. In order to reach nanodimensions, size reduction is iterated using the outcome of each preceding step in subsequent drawing steps. The nanostructures obtained by the new fabrication method; direct-size reduction [1] are unique in their optical features.

Particularly, we have investigated core-shell nanostructures which offer outstanding attributes in color formation by enhancing tunability in the physical properties, which is apparently limited in many other one dimensional nanostructures, regarding unprecedented material characteristics of composite structures and flexibility in the design. They exhibit and are suited for both interference based and small particle scattering based coloring [2]. Core shell nanowires are produced starting from a macroscopic $\mathrm{As}_{2} \mathrm{Se}_{3}$ glass rod, wrapped in a polyvinylidene fluoride (PVDF) polymer sheet, and consolidated in a polyethersulfone (PES) jacket. PES is used for keeping whole structure together during thermal drawing, later to be removed in order to obtain free-standing core-shell nanowires, if required. Thermal drawing of the preform is carried out by controlling tension, temperature, feed-in, and drawing speed in a custom-built fiber tower optimized for nanowire production. After the first drawing step, we obtained $\mathrm{As}_{2} \mathrm{Se}_{3}$ microwires embedded in a PES fiber with total diameter of $400 \mu \mathrm{m}$. Afterward, we cut a certain number of fibers obtained from the first step, stacked, embedded in a PES jacket, and consolidated them for a second drawing step. After the second thermal drawing, we obtained core-shell nanowires of 70-1800 nm diameters by altering the reduction factor. By repeating this procedure for the third time, we produced core-shell nanowires with diameters down to $10 \mathrm{~nm}$. This method ensures us to obtain flexible, polymer embedded, indefinitely long, ordered, uniform core-shell nanowires with precise control of physical and geometrical properties and chemical composition. These core-shell nanowires have two characteristic radial size scales that give rise to respective coloration mechanisms. Simply by altering drawing conditions, i.e. changing the reduction factor, any hue from the visible spectrum can be effectively obtained (Fig. 1a). High refractive index chalcogenide glasses enhance interference effects and increase scattering efficiency at nano dimensions. 
Both coloring mechanisms could cover full visible spectrum by arranging nanoshell thickness and core diameter respectively. Nanoshell coloration becomes apparent when the shell thickness is in the range of 160-280 $\mathrm{nm}$. As the core diameter reduce to smaller sizes $(200-30 \mathrm{~nm})$, covering shell thicknesses reduce simultaneously and become extremely thin. Interference effect due to the covering shell layers is no longer observable for these coreshell nanowires, but it is now replaced by a new coloration mechanism (Fig. 1b-e) originated from resonant Mie scattering. The core diameter determines the structure and the number of the modes resonating inside the nanowire, which could be described as of whispering gallery type.

a.

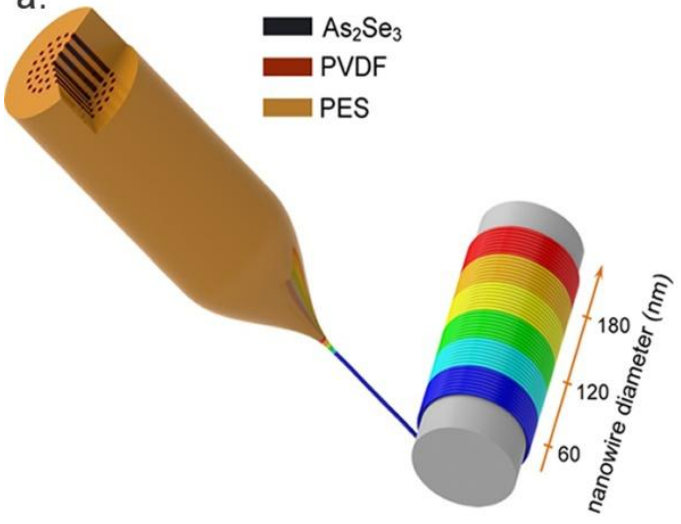

b.

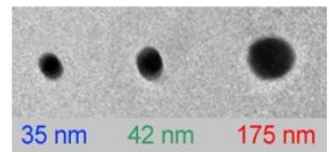

c.

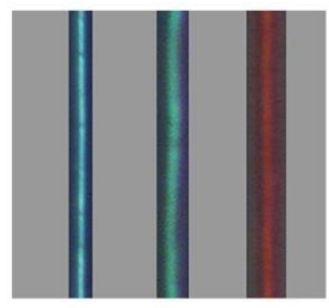

d.

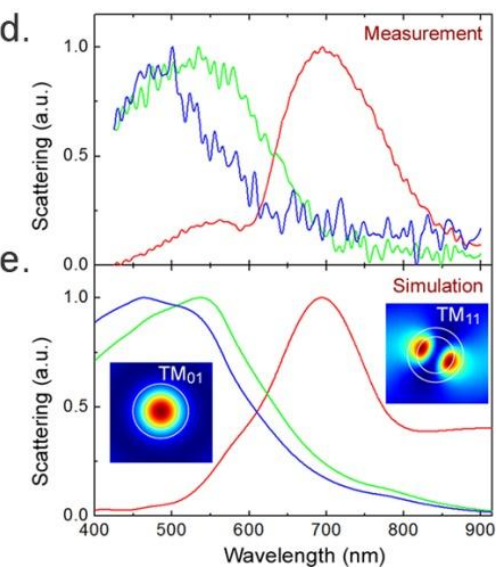

Figure 1. (a) Fiber embedded nanowire structure is thermally drawn from a composite that has an arsenic selenide core, an isolation polymer layer (poly vinyl fluoride) and a wrapping second jacket polymer (poly ether sulphone). Diameter dependent coloration obtained during drawing process. Characterization of Mie scattering based coloration. (b) Cross-sectional TEM images of nanostructures. (c) Colored nanowire images taken by optical microscope. (d) Measurement and (e) Simulation are good agreement with each other.

Polymer fiber embedded one-dimensional nanostructures can be used singly or collectively, using array based coloration or photonic crystal based coloration, as iridescent or solid color centers. In addition to textile and decoration applications, nanostructures can be used as functional modules as solar cell concentrators, as stimuli responsive polymers, and in biological imaging, providing unique applications. Using this fabrication methodology integration of nanowires into photodetection circuitry [3] also demonstrated recently. The 2D and 3D lattice of nanowires may exhibit negative index of refraction at optical domain and coupled core-shell nanowire arrays can be used to generate slow light.

\section{References}

[1] M. Yaman, M. Bayindir et al., “Arrays of indefinitely-long, uniform nanowires and nanotubes,” Nature Materials 10, 494-501 (2011).

[2] T. Khudiyev, M. Bayindir et al., "Structural Coloring in Large Scale Core-Shell Nanowires," Nano Letters, 11, 4661-4665, (2011).

[3] E. Ozgur, M. Bayindir et al., "Macroscopic Assembly of Indefinitely Long and Parallel Nanowires into Large Area Photodetection

Circuitry," Nano Letters 12, 2483-2487 (2012). 